\title{
Fas-mediated apoptosis and peripheral polyneuropathy in type 2 diabetes mellitus
}

\author{
Mowaffak M. Abd Elhameed ${ }^{a}$, Noha A. Elsawy ${ }^{a}$, Magdy H. Zekry Mgalaa ${ }^{b}$, \\ Sarah S. El-Tawab ${ }^{a}$, Reham A. Abo Elwafac ${ }^{c}$, Marwa R. Abd Elfadeel ${ }^{\mathrm{a}}$
}

Departments of aphysical Medicine, Rheumatology and Rehabilitation, ${ }^{b}$ Internal Medicine, ${ }^{\circ}$ Clinical and Chemical Pathology, Faculty of Medicine, Alexandria University, Alexandria, Egypt

Correspondence to Marwa R. Abd Elfadeel, Master Degree of Physical Medicine, Rheumatology and Rehabilitation, Medaan El-Khartoom Square, Al-Azaritah, Alexandria 02030, Egypt. Tel: +20 100860 0356; e-mail: marwarabi3abdelfadel@outlook.com

Received 23 April 2019

Accepted 23 May 2019

Egyptian Rheumatology \& Rehabilitation 2019, 46:321-326

\begin{abstract}
Objective different diabetic factors among those patients.

Patients and methods sFas and sFasL were assessed in all the studied groups.

\section{Results} difference between all the studied groups.

\section{Conclusion} and is correlated with its electrophysiological severity.

\section{Keywords:}

distal symmetrical polyneuropathy, soluble Fas ligand, soluble Fas

Egypt Rheumatol Rehabil 46:321-326

(c) 2019 Egyptian Society for Rheumatology and Rehabilitation

1110-161X
\end{abstract}

To evaluate the role of soluble Fas (sFas) and soluble Fas ligand (sFasL) in the pathogenesis of distal symmetrical polyneuropathy (DSPN) in patients with type 2 diabetes mellitus, and to analyze the relationship between these apoptotic markers with clinical parameters and electrophysiologic profile of DSPN, as well as with

The study included 60 Egyptians with type 2 diabetes mellitus. All patients were evaluated clinically for DSPN by using Michigan Neuropathy Screening Instrument. Electrophysiological diagnosis of DSPN was based on the criteria suggested by the European Standardized Telematic tool to Evaluate Electrodiagnostic Methods group. Diabetic patients were divided into two groups according to the electrophysiological findings: group A included patients with DSPN $(N=42)$, and group B included patients without DSPN $(N=18)$. The severity of DSPN among group A patients was assessed clinically using Toronto Clinical Neuropathy Score and electrophysiologically by the severity score proposed by Hidasi and colleagues. The study also included 30 healthy volunteers as a control group. Serum levels of

Serum level of sFas was significantly elevated in diabetic patients with DSPN compared with diabetics without DSPN and nondiabetic control $(P=0.029$ and 0.000$)$. Receiver operating characteristic (ROC) curve analysis detected that sFas was statistically significant in discriminating between diabetic patients with DSPN from those patients without DSPN with an accuracy of $66 \%$. The cutoff point that has the highest sensitivity $(61 \%)$ and specificity $(62 \%)$ was $33.3 \mathrm{ng} / \mathrm{ml}$. Serum level of sFas showed a positive significant correlation with the electrophysiological severity of DSPN $(P=0.020)$. Serum level of $\mathrm{sFasL}$ did not show statistically significant

Fas-mediated apoptosis has an important role in the development of diabetic DSPN

\section{Introduction}

Diabetes mellitus (DM) is a public disease with rapidly increasing rates worldwide [1]. The prevalence of type $2 \mathrm{DM}$ is $\sim 90 \%$ of people with diabetes around the world [2]. It is estimated that by the year 2030, Egypt will have at least 8.6 million adults with diabetes [3]. Diabetic peripheral neuropathy (DPN) is the most prevalent and troublesome complication in patients with DM [4,5]. Distal symmetrical polyneuropathy (DSPN) is the commonest, accounting for $75 \%$ of diabetic neuropathy [6]. Despite the advances in methods to control blood glucose level, the development of DPN is probably neither preventable nor controllable [7].
Discussing the pathogenesis of DPN includes various factors [8]. Although hyperglycemia is considered to be the major pathophysiological factor in the development of DPN, indeed the associated mechanisms need to be clarified. Apoptosis is one of these mechanisms that may play a crucial role in the pathogenesis of DPN [9]. Several apoptotic pathways have been described. One of those is the Fas system, which is believed to be the first initiator of apoptosis. It is composed of Fas ligand

\footnotetext{
This is an open access journal, and articles are distributed under the terms of the Creative Commons Attribution-NonCommercial-ShareAlike 4.0 License, which allows others to remix, tweak, and build upon the work non-commercially, as long as appropriate credit is given and the new creations are licensed under the identical terms.
} 
(FasL), a type II transmembrane glycoprotein, and Fas antigen (Fas/Apo-1/CD95), a type I transmembrane glycoprotein receptor. Cross-linking of Fas by FasL triggers apoptosis in various target cells [10]. Fas is widely expressed in the nervous system in both neurons and glial cells [11]. The increased production of soluble Fas (sFas) and soluble Fas ligand (sFasL) proteins indicates a sFas/sFasL interaction in patients with DPN [12]. The rise of $\mathrm{sFas}$ could be by overexpression of Fas receptor by peripheral nerve cells. Free oxygen radicals, caspase 8, and growth factors could all regulate Fas expression in nervous system [13].

Although the Fas-mediated apoptosis in DSPN has been discussed previously in the literature, its relation to different factors has to be studied more precisely. The aim of this study was to discover the Fas-mediated apoptosis in DSPN in type 2 diabetic patients and to correlate it with the clinical and electrophysiological severity of DSPN, as well as with different diabetic factors among those patients.

\section{Patients and methods}

The study included 60 Egyptians with type 2 DM according to the American Diabetes Association Guidelines [14]. Patients were recruited randomly from those attending the Outpatient Clinics of Physical Medicine, Rheumatology and Rehabilitation Department, Alexandria University Hospital. Patients who had peripheral neuropathy (PN) with principal causes other than diabetes such as liver or renal disease, toxic exposure, inflammatory diseases, and endocrine, metabolic, nutritional, or malignant disorders were excluded from the study. Patients with diabetic neuropathy with other forms than distal sensory, motor, or sensorimotor polyneuropathy were also excluded from the study.

All patients were evaluated clinically for DSPN by using Michigan Neuropathy Screening Instrument questionnaire and physical examination [15]. The standard electrophysiological study was done for all patients [16]. All procedures were carried out on Neuropack 2 electromyograph apparatus from Nihon Kohden (Tokyo, Japan). Electrophysiological diagnosis of DSPN was based on criteria for diagnosis of polyneuropathy suggested by the European Standardized Telematic tool to Evaluate Electrodiagnostic Methods group [17]. The same set of criteria was used to define the prevalent pathology among all patients with DSPN [17]. Diabetic patients were divided according to the electrophysiological findings into two groups: group $\mathrm{A}$ included diabetic patients with DSPN $(N=42)$, and group B included diabetic patients without DSPN $(N=18)$. The study also included 30 healthy volunteers as a control group with matching age, sex, and general constitution. The severity of DSPN among group A patients was assessed clinically using Toronto Clinical Neuropathy Score (TCNS) [18]. In the TCNS, clinical severity of DPN was scored by a numerical value ranging from 0 to 19 points, which was calculated by summing symptom score points (the presence or absence of foot pain, numbness, tingling, weakness, ataxia, and upper limb symptoms), reflex score points (bilateral knee and ankle reflexes, each graded as absent, reduced, or normal), and physical examination score points (the presence or absence of pinprick, temperature, light touch, vibration, and position sense) [18]. Electrophysiological severity of DSPN was assessed according to the score proposed by Hidasi et al. [19], according to which the electrophysiologic parameters of eleven segments were measured (two segment of the motor fibers of ulnar and peroneal nerves, and one segment of the motor fibers of the median nerve and of the sensory fibers of the median, ulnar, and sural nerves). The distal latency of peroneal nerves and the conduction velocities and amplitudes of the motor and the sensory potentials were assessed. The quantitative electrophysiological abnormalities in each nerve were assessed using electrophysiologic nerve score for evaluation of the degree of the peripheral neuropathy in each nerve. Assessment of DM control was done using glycated hemoglobin (HbA1c) by immunoinhibition method [20]. Serum levels of $s F a$ and $s F a s L$ were measured by enzyme-linked immunosorbent assay method [21].

Written consent, which was approved by the Alexandria Ethical Committee, was obtained from all patients after a full explanation of the study.

\section{Statistical methods}

Data were analyzed using the statistical package for the social sciences (SPSS version 20; SPSS Inc., Chicago, Illinois, USA). The distributions of quantitative variables were tested for normality using Kolmogorov-Smirnov test. Normally distributed data were described using mean and SD, whereas nonnormally distributed data were described using median and range. Qualitative data were described using number and percent. One-way analysis of variance test was used to compare parametric variables between more than two groups, followed by Games-Howell post-hoc test if significant, whereas Kruskal-Wallis test was used to compare nonparametric data, followed by pairwise 
comparisons if significant. Comparison of quantitative normally distributed variables between two groups was done using independent samples $t$-test, and Mann-Whitney $U$-test was used for nonparametric data. Pearson $\chi^{2}$ was used to compare $2 \times 2$ categorical variables, and Fisher's exact test when if more than $20 \%$ of cells had expected cell count less than 5. Spearman rank correlation test was used to correlate between two quantitative groups. In all statistical tests, level of significance of 0.05 was used, below which the results were considered to be statistically significant.

\section{Results}

The mean age was $50.9 \pm 7.2$ years in group A, 51.16 \pm 6.89 years in group $B$, and $51.06 \pm 7.74$ years in group C. Most diabetic patients with and without DSPN were female (66.7 and $72.2 \%$, respectively). The mean BMI was $31.3 \pm 5.7 \mathrm{~kg} / \mathrm{m}^{2}$ in group A, $30.4 \pm 6.14 \mathrm{~kg} / \mathrm{m}^{2}$ in group B, and $28.5 \pm 2.1 \mathrm{~kg} / \mathrm{m}^{2}$ in group C. Group A patients had a statistically significant longer duration of diabetes as compared with patients of group B (12.8 \pm 5.77 vs. $4.1 \pm 2.26$ years, $t=3.6$ at $P=0.001)$. The $\mathrm{HbA1c}$ level was significantly higher in group A patients than in group B patients $(9.73 \pm 2.32$ vs. 7.7 $\pm 1.5 \%$ and $P=0.001$ ). Based on the results of TCNS, most of our patients had moderate and severe DSPN (76.3\%), whereas $23.7 \%$ had mild DSPN. Results of the electrophysiologically assessed severity score constructed by Hidasi et al. [19] revealed that $67 \%$ of group A patients had moderate and severe DSPN, whereas 33\% had mild DSPN. Axonal degeneration was the most prevalent pathology among patients with DSPN included in the study (60\%), whereas only $9 \%$ of the patients had demyelinating pathology, 19\% had mixed pathology, and the remaining $12 \%$ of the patients were considered neuropathic and could not be classified into any of the previous categories. There were significant positive correlations between both diabetes duration and $\mathrm{HbA} 1 \mathrm{c}$ level with clinical severity, assessed by TCNS, and electrophysiologic severity of DSPN $(P<0.000$; Table 1$)$.

Serum level of sFas was significantly elevated in group A patients as compared with groups $\mathrm{B}$ and $\mathrm{C}(P=0.029$ and 0.000 , respectively). sFas serum level was significantly high in group B when compared with group $\mathrm{C}(P=0.000$; Table 2$)$. On the contrary, the mean value of sFasL serum levels did not show any statistically significant difference between all the studied groups (Table 2). The receiver operating characteristic (ROC) curve analysis of $s F a s$ was statistically significant in discriminating between diabetic patients with DSPN from those patients without DSPN with an accuracy of $66 \%$. The cutoff point which has the highest sensitivity (61\%) and specificity (62\%) was $33.3 \mathrm{ng} / \mathrm{ml}$ (Fig. 1). Serum level of sFas showed a positive significant correlation with the electrophysiological severity of DSPN $\left(r_{\mathrm{s}}=0.359\right.$ at $\left.P=0.020\right)$. On the contrary, its level lacked significant correlation with clinical severity assessed by TCNS (Table 3). In addition, there were no significant correlations between serum levels of sFasL with either clinical or electrophysiological severity of DSPN (Table 3). Serum level of sFas showed a significant positive correlation with HbA1c level among group A patients $\left(r_{\mathrm{s}}=0.325\right.$ at

Table 1 Correlation between clinical and electrophysiological severity of distal symmetrical polyneuropathy with diabetes duration, and glycated hemoglobin, among group A patients

\begin{tabular}{lcc}
\hline $\begin{array}{l}\text { Group A (DM } \\
+\mathrm{DSPN})\end{array}$ & $\begin{array}{c}\text { Eletrophysiologically assessed } \\
\text { severity score }\end{array}$ & Toronto \\
\hline $\begin{array}{l}\text { DM duration } \\
\text { (years) }\end{array}$ & $r_{\mathrm{s}=0.592}$ & $r_{\mathrm{s}=0.745}$ \\
& $P=0.000^{*}$ & $P=0.000^{*}$ \\
$\mathrm{HbA} 1 \mathrm{c} \%$ & $r_{\mathrm{s}=0.514}$ & $r_{\mathrm{s}=0.553}$ \\
& $P=0.001^{*}$ & $P=0.000^{*}$ \\
\hline
\end{tabular}

DM, diabetes mellitus; DSPN, distal symmetrical polyneuropathy; HbA1c, glycated hemoglobin; $r_{\mathrm{s}}$, Spearman's $\rho$ test. ${ }^{*} P \leq 0.05$, statistically significant.

Table 2 Analysis of variance of serum levels of soluble Fas and soluble Fas ligand Serum level among the studied groups

\begin{tabular}{|c|c|c|c|c|c|c|c|}
\hline & $\begin{array}{l}\text { Group A DM } \\
+ \text { PN }(n=42)\end{array}$ & $\begin{array}{c}\text { Group B DM- } \\
\text { PN }(n=18)\end{array}$ & $\begin{array}{c}\text { Group C } \\
\text { control }(n=30)\end{array}$ & $F$ & $P$ & $\begin{array}{c}\text { Post hoc } \\
\text { In-between group } \\
\text { comparison }\end{array}$ & $P$ \\
\hline \multicolumn{8}{|l|}{ sFas (ng/ml) } \\
\hline Minimum-maximum & $10.75-69.12$ & $17.31-56.74$ & $3.91-22.5$ & 53 & $0.001^{*}$ & $\begin{array}{c}A \text { and } B A \text { and } C B \\
\text { and } C\end{array}$ & $0.029^{\star} 0.000^{\star} 0.000^{\star}$ \\
\hline Mean \pm SD & $40.96 \pm 17.7$ & $31.12 \pm 10.73$ & $13.14 \pm 6.7$ & & & & \\
\hline \multicolumn{8}{|l|}{ sFasL (ng/ml) } \\
\hline Minimum-maximum & $0.04-0.2$ & $0.03-0.17$ & $0.04-0.17$ & 0.054 & 0.94 & & \\
\hline Mean \pm SD & $0.10 \pm 0.045$ & $0.098 \pm 0.046$ & $0.097 \pm 0.042$ & & & & \\
\hline
\end{tabular}

$\mathrm{DM}$, diabetes mellitus; PN, peripheral neuropathy; $F$, one-way analysis of variance post hoc Games-Howell test; sFas, soluble Fas; sFasL, soluble Fas ligand. ${ }^{*} P \leq 0.05$, statistically significant. 
Fig. 1

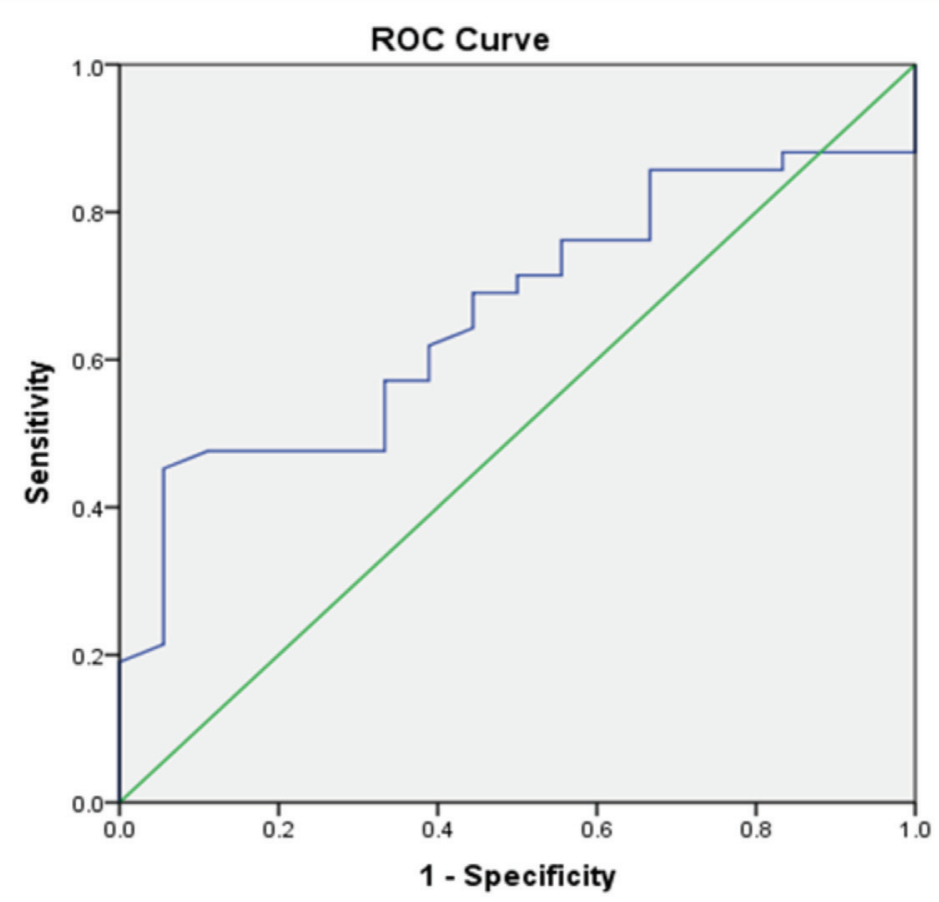

Diagonal segments are produced by ties.

ROC curve of soluble Fas serum level.

Table 3 Correlation between clinical and electrophysiological severities of distal symmetrical polyneuropathy with soluble Fas and soluble Fas ligand

\begin{tabular}{lccccc}
\hline Group A & \multicolumn{2}{c}{$\begin{array}{c}\text { Eletrophysiologically- } \\
\text { assessed severity } \\
\text { score }\end{array}$} & & \multicolumn{2}{c}{ TCNS } \\
\cline { 2 - 3 } \cline { 5 - 6 } & $r_{\mathrm{s}}$ & $P$ & & $r_{\mathrm{s}}$ & $P$ \\
\hline sFas & 0.359 & $0.020^{*}$ & & 0.194 & 0.218 \\
sFasL & -0.189 & 0.230 & & 0.252 & 0.107 \\
\hline
\end{tabular}

$r_{\mathrm{s}}$, Spearman's $\rho$ test; sFas, soluble Fas; sFasL; soluble Fas ligand; TCNS, Toronto Clinical Neuropathy Score. ${ }^{*} P \leq 0.05$, statistically significant.

Table 4 Correlation between serum level of soluble Fas with diabetes duration and glycated hemoglobin among group A patients

\begin{tabular}{lcc}
\hline Group A (DM+DSPN) & DM duration (years) & HBA1c\% \\
\hline sFas & $r_{\mathrm{s}=0.190}$ & $r_{\mathrm{s}=0.325}$ \\
$P$ & 0.228 & $0.035^{*}$ \\
\hline
\end{tabular}

DM, diabetes mellitus; DSPN, distal symmetrical polyneuropathy; HBA1c, glycated hemoglobin; $r_{\mathrm{s}}$, Spearman's $\rho$ test; sFas, soluble Fas. ${ }^{*} P \leq 0.05$, statistically significant.

$P=0.035)$; however, it did not show significant correlation with diabetes duration (Table 4).

\section{Discussion}

The role of Fas-mediated apoptosis in the development of DSPN was suggested previously in the literature. The aim of this study was to evaluate this role and further analyze the possible connection between this pathway and both clinical parameters and the electrophysiological profile of DSPN in patients with type $2 \mathrm{DM}$, as well as with different diabetic factors.

In this study, diabetic patients with DSPN had statistically significant longer diabetes duration, and higher HbA1c level than those without DSPN. These results run in accordance with many of the DSPN prevalence studies carried out across the world, in which poor glycemic control and longer duration of diabetes were identified as risk factors for DPN [22-24]. Moreover, there were significant positive correlations between both diabetes duration and $\mathrm{HbA1c}$ level with clinical severity, assessed by TCNS, and electrophysiologic severity of DSPN $(P<0.000)$ These detected correlation adds more importance on the duration of diabetic illness and the glycemic control over years for the development of DSPN. Similar results were reported by Kamel et al. [25] and Kovac et al. [26].

This study revealed a significant increase in the mean value of sFas serum level in diabetic patients with DSPN compared with diabetic patients without DSPN $(P=0.028)$ and control group $(P=0.000)$. Increased serum concentrations of $\mathrm{sFas}$ are considered to reflect activation of the Fas/FasL system, which is an important inducer of apoptosis. Moreover, the significant increase 
of sFas serum level in diabetic peripheral neuropathy patients indicates that Fas-mediated apoptosis may have a role in the etiopathogenesis of DSPN. Our findings were similar to those reported by previous studies $[12,27]$. Furthermore, we demonstrated in our study by ROC curve analysis that sFas was statistically significant in discriminating between diabetic patients with DSPN from those patients without DSPN, with an accuracy of $66 \%$. The cutoff point that has the highest sensitivity (61\%) and specificity (62\%) was $33.3 \mathrm{ng} / \mathrm{ml}$. This result suggests the use of serum $\mathrm{sFas}$ level as a laboratory marker in the detection of DSPN. The present study showed a positive significant correlation between serum level of $\mathrm{sFas}$ and electrophysiological assessed severity score $(P=0.020)$, supporting the involvement of sFas in the advancement of DPN [13]. Our findings agree with the findings of Zakareia [27] and Al-Rouq [12], who reported a significant correlation between serum level of $\mathrm{sFas}$ with deterioration of different electrophysiological parameters. On the contrary, we found insignificant correlation between sFas serum level and clinical severity of DSPN assessed by TCNS $(P=0.218)$. This discrepancy between clinical findings and electrophysiological results with the serum sFas could be explained by the symptomatic part of the TCNS making its total score partially subjective compared with the accurate results of NCS. To the best of our knowledge, no previous study assessed the correlation between serum level of $\mathrm{sFas}$ and the clinical severity.

Despite the significant difference of serum level of sFas we found between our studied groups, the mean value of sFasL serum levels did not show any statistically significant difference between all the studied groups $(P=0.94)$, running with previously reported studies $[12,21]$, which was explained by a-sFasL might be cross-linked with $\mathrm{Fas}$ or $\mathrm{sFas}$, and consequently, its free soluble form not available in the serum [21]. The antibodies used for detection of sFasL might not recognize the $s F a s L$ epitope present in the sera from diabetic patients and normal controls. Criticism about the detection of cell surface FasL suggests that antibodies used to recognize FasL were not always appropriate for the detection of this protein [28]. cFas-mediated apoptosis might be independent from the Fas-FasL interaction, as observed in hepatocytes where it has been reported that bile salts induce apoptosis via direct activation of Fas [29]. Accordingly, no significant correlation between serum level of sFasL with neither clinical or electrophysiological severity could be found in this study as its level was normal in the three studied groups ( $P=0.107$ and 0.230 , respectively).
Contrary to our results, Mondal et al. [30] reported highly significant decrease in sFasL level in patients with DSPN compared with diabetic patients without DSPN and healthy controls. Moreover, they reported a significant negative correlation between serum level of sFasL and clinical severity of DSPN assessed using vibration perception threshold. They suggested that the enhanced apoptotic activity in DSPN may be owing to increased interaction between upregulated $\mathrm{sFas}$ with overexpressed membrane-bound FasL at conserved cleavage site with a lesser cleavage to generate sFasL, which may explain the decrease of serum level of sFasL in patients with DSPN.

In this study, serum level of sFas showed a significant positive correlation with $\mathrm{HbA1c}$ level among diabetic patients with DSPN $\left(r_{\mathrm{s}}=0.325\right.$ at $\left.P=0.035\right)$; however, it did not show significant correlation with diabetes duration. Similar findings were reported by Hamid et al. [31]. Increased glucose concentration was reported to induce apoptosis directly through upregulation of Fas receptors [32]. However, longer diabetes duration does not always reflect uncontrolled hyperglycemia through all this duration. This may explain the absence of significant correlation between serum level of $\mathrm{sFas}$ and diabetes duration. In addition, serum level of sFas was significantly elevated in diabetic patients with DSPN, and this duration represents the duration of type $2 \mathrm{DM}$ not the duration of DSPN.

\section{Conclusion}

Fas-mediated apoptosis has an important role in development of diabetic DSPN and is correlated with its electrophysiological severity.

\section{Financial support and sponsorship}

Nil.

\section{Conflicts of interest}

There are no conflicts of interest.

\section{References}

1 Maruthur NM. The growing prevalence of type 2 diabetes: increased incidence or improved survival? Curr Diab Rep 2013; 13:786-794.

2 World Health Organization (WHO). Global status report on noncommunicable diseases 2010. Geneva: WHO; 2011.

3 Shaw JE, Sicree RA, Zimmet PZ. Global estimates of the prevalence of diabetes for 2010 and 2030. Diabetes Res Clin Pract 2010; 87:4-14.

4 Seung HK, Yun Cha B. Diabetic peripheral neuropathy in type 2 diabetes mellitus in Korea. Diabetes Metab J 2012; 36:6-12.

5 Manouk B, Charles A. Prevalence and complications of diabetes mellitus in Northern Africa, a systematic review. BMC Public Health 2013; 13:387.

6 Smith SC, Lamping DL, Maclaine GD. Measuring health-related quality of life in diabetic peripheral neuropathy: a systematic review. Diabetes Res Clin Pract 2012; 96:261-270. 
7 Shi X, Chen Y, Nadeem L, Xu G. Beneficial effect of TNF- $\alpha$ inhibition on diabetic peripheral neuropathy. J Neuroinflammation 2013; 10:69.

8 Hinder LM, Vincent AM, Burant CF, Pennathur S, Feldman EL. Bioenergetics in diabetic neuropathy: what we need to know. J Peripher Nerv Syst 2012; 17:10-14.

9 Cosson E, Bringuier AF, Paries J, Guillot R, Vaysse J, Attali JR, et al. Fas/ Fas-ligand pathway is impaired in patients with type 2 diabetes. Influence of hypertension and insulin resistance. Diabetes Metab 2005; 31:47-54.

10 Nat R, Radu E, Regalia T, Popescu LM. Apoptosis in human embryo development: Fas induced apoptosis in brain primary cultures. J Cell Mol Med 2001; 5:417-428.

11 Toyozaki T, Hiroe M, Saito T. Levels of soluble Fas in patients with myocarditis, heart failure of unknown origin, and in healthy volunteers. Am J Cardiol 1998; 81:798-800.

12 Al-Rouq F. Nerve conduction study and Fas mediated apoptosis of nerve cells in peripheral neuropathy in type 2 diabetes. Int J Diab Mellitus 2009; 1:38-39.

13 Wernig F, Xu Q. Mechanical stress-induced apoptosis in the cardiovascular system. Prog Biophys Mol Biol 2002; 78:105-137.

14 Diagnosis and Classification of Diabetes Mellitus. American Diabetes Association. Diabetes Care 2014; 37:81-90.

15 Mete T, Aydin Y, Saka M, Yavuz HC, Bilen S, Yalcin Y, et al. Comparison of efficiencies of Michigan neuropathy screening instrument, neurothesiometer, and electromyography for diagnosis of diabetic neuropathy. Int J Endocrinol 2013; 2013:821745.

16 Oh SJ. Clinical electromyography: nerve conduction studies. Balitmore: University Park Press; 1984.

17 Tankisi H, Pugdahl K, Fuglsang-Frederiksen A, Johnsen B, de Carvalho M, Fawcett PR, et al. Pathophysiology inferred from electrodiagnostic nerve tests and classification of polyneuropathies. Suggested guidelines. Clin Neurophysiol 2005; 116:1571-1580.

18 Bril V, Perkins BA. Validation of the Toronto Clinical Scoring System for diabetic polyneuropathy. Diabetes Care 2002; 25:2048-2052.

19 Hidasi E, Dioszeghy P, Fulesdi B, Mechler F. Electroneurographic examinations in insulin- and non-insulin dependent diabetes mellitus. Electroencephalogr Clin Neurophysiol 1996; 99:303.

20 Knowles WJ, Haigh WB. Amonoclonal antibody-based immunoassay for HbA1c. Diabetes 1986; 35:94.
21 Guillot R, Bringuier AF, Porokhov B, Guillausseau PJ, Feldmann G. Increased levels of soluble Fas in serum from diabetic patients with neuropathy. Diabetes Metab 2001; 27:315-321.

22 Nisar M, Asad A, Waqas A, Ali N, Nisar A, Qayyum MA, et al. Association of diabetic neuropathy with duration of type 2 diabetes and glycemic control. Cureus 2015; 7:e302.

23 Kasim K, Amar M, ElSadek AA, Abdel Gawad S. Peripheral neuropathy in type-II diabetic patients attending diabetic clinics in Al-Azhar University Hospitals, Egypt. Int J Diab Mellitus 2009; 2:20-23.

24 Abougalambou SS, Abougalambou AS. Explorative study on diabetes neuropathy among type II diabetic patients in Universiti Sains Malaysia Hospital. Diab Metab Syndr 2012; 6:167-172.

25 Kamel SR, Hamdy M, Abo Omar HA, Kamal A, Ali LH, Abd Elkarim AH. Clinical diagnosis of distal diabetic polyneuropathy using neurological examination scores: correlation with nerve conduction studies. Egypt Rheumatol Rehabil 2015; 42:128-136.

26 Kovac B, Emedi SM, Svalina S, Demarin V. Clinical and electrophysiological signs of diabetic polyneuropathy, the effect of glycemia and duration of diabetes mellitus. Acta Clin Croat 2011; 50:149-157.

27 Zakareia FA. Electrophysiological changes, plasma vascular endothelial growth factor, fatty acid synthase, and adhesion molecules in diabetic neuropathy. Neurosciences 2008; 13:374-379.

28 Smith D, Sieg S, Kaplan D. Technical note: aberrant detection of cell surface Fas ligand with anti-peptide antibodies. J Immunol. 1998; 160:4159-4600.

29 Faubion WA, Guicciardi ME, Miyoshi H, Bronk SF, Roberts PJ, Svingen PA, et al. Toxic bile alts induce rodent hepatocyte apoptosis via direct activation of Fas. J Clin Invest. 1999; 103:137-145.

30 Mondal A, Sen S, Chanda D, kundu S, Chatterjee M, Mukherjee S. Evaluation of diabetic polyneuropathy in type 2 diabetes mellitus by nerve conduction study and association of severity of neuropathy with serum sFasL level. Indian J Endocrinol Metab 2012; 16:465-467.

31 Hamid SM, Shani WS. Association of sFas and sFas ligand with progression of type 2 diabetes mellitus in Basrah province. Med $\mathrm{J}$ Babylon 2018; 15:135-138.

32 Maedler K, Spinas G, Lehmann R, Sergeev P, Weber M, Fontana A, et al. Glucose induces b-cell apoptosis via upregulation of the fas receptor in human islets. Diabetes 2001; 50:1683-1690. 